\title{
Sistema de control automatizado en planta de cargue de agua potable para camiones cisterna
}

\section{Automated control system in drinking water plant for loading of tankers}

\author{
Faiber Ignacio Robayo ${ }^{[1]}$, Dayana Melisa Silva P. ${ }^{[2]}$ y David Julián Mosquera A. ${ }^{[3]}$
}

\begin{abstract}
Resumen
Este proyecto consiste en el diseño y la implementación de un sistema de control computarizado para la automatización de la planta "La Esmeralda” ubicada en las afueras del norte de la ciudad de Neiva, en la cual se realiza el cargue de agua potable para camiones cisterna. En ella se hace la instalación de un conjunto de instrumentos electrónicos para tomar el control de las bombas y las válvulas del tanque de almacenamiento y se realiza el arreglo necesario para la ubicación y el montaje del sensor ultrasónico, esencial en el monitoreo de la planta. Por lo tanto se monitorea la apertura y cierre de las válvulas, el encendido y apagado de las bombas sumergibles y el nivel del tanque en tiempo real por medio de una interfaz gráfica LabVIEW que a su vez permite tomar los datos del cliente quien hace un requerimiento del servicio y guardarlos en una base de datos. Como resultado de este trabajo, se obtiene una planta completamente automatizada capaz de suministrar de forma automática los volúmenes de agua requeridos por el cliente, todo controlado por medio de una aplicación en LabVIEW que permite monitorear y visualizar en tiempo real los acontecimientos que genera el proceso de cargue de agua potable a los camiones cisterna.
\end{abstract}

Palabras clave: Control, automatización, instrumentación, tiempo real, LabVIEW.

\begin{abstract}
This project involves the design and implementation of a computerized control system for the automation of the "La Esmeralda" plant located in the city of Neiva, where drinking water is loaded onto tankers. A set of electronic instruments is installed to control the pumps and valves of the storage tank, and the necessary adjustments are made for the location and mounting of the ultrasonic sensor, essential for plant monitoring. The sensor monitors the opening and closing of valves, the turning on and off of the submersible pumps, and tank levels in real time, through the implementation of a LabVIEW graphical interface. This, in turn, allows the recording of customer data, which is then stored in a database. As a result of this work, a fully automated plant capable of supplying automatic water volumes required by the customer is obtained, controlled through a LabVIEW application that allows real-time monitoring and visualization of events that occur during the loading of drinking water onto the tankers.
\end{abstract}

Key words: Control, automation, instrumentation, real time, LabVIEW.

[1] Magister en Ingeniería de Control. Docente Universidad Surcolombiana. Avenida Pastrana Carrera 1ª faiber.robayo@ usco.edu.co

[2] Ingeniero Electrónico. Universidad Surcolombiana Neiva. Avenida Pastrana Carrera 1a. u2007269511@usco.edu.co

[3] Ingeniero Electrónico. Universidad Surcolombiana Neiva. Avenida Pastrana Carrera 1ª u2008172463@usco.edu.co

Recibido: 1 marzo 2015 • Aprobado: 2 abril 2015 


\section{Introducción}

La importancia de la automatización en la industria de procesos ha aumentado increíblemente en los últimos años $y$, de hecho, se ha convertido en una fuerza impulsora de todo el sector químico, petrolero, gasífero y biotecnológico. Sistemas de instrumentación innovadores controlan procesos complejos, garantizan la fiabilidad y seguridad de los procesos, y son la base de estrategias avanzadas de mantenimiento (Magazine Química, 2009). Los avances tecnológicos han incidido fuertemente en el aumento de la productividad y aprovechamiento de los recursos, pudiéndose afirmar que casi el $100 \%$ de la producción industrial mundial es controlada por sistemas de controles automáticos o semiautomáticos (Electroindustria, 2014). La automatización es un proceso donde las tareas de producción que son realizadas por humanos se transfieren a las máquinas para aplicar el uso de la tecnología (QuimiNet, 2012).

Durante casi tres décadas, ingenieros y científicos han utilizado y confiado en el software de diseño de sistemas para adquirir, analizar y visualizar los datos del mundo real (Electrónica, 2014). La interface hombre-máquina o estación de operación, es la estación a través de la cual el operador de sala de control observa y toma acción sobre el control regulatorio de su proceso productivo, arranca y para equipos, visualiza alarmas y gráficas de tendencia de las diferentes variables (Dominguez, 2007). Para lograr un buen funcionamiento de los proceso es necesario mantener permanentemente los equipos e instalaciones, en su mejor estado para evitar los tiempos de parada que aumentan los costos (Atlantic International University, 2008).

La realización de este proyecto está orientada a la implementación de un sistema de control computarizado donde se obtiene el monitoreo, registro y visualización de un conjunto de elementos y variables de una planta de cargue de agua potable para camiones cisterna. Se controlan distintas variables y que suministran la cantidad necesaria y exacta de agua potable que se transporta por medio de camiones cisterna a varios sectores de la ciudad de Neiva; siendo ellos los clientes que compran el líquido con un fin determinado.

El propósito del desarrollo de este proyecto no solo trae beneficios económicos al propietario de la planta La Esmeralda y a sus clientes, si no también, ayuda al desarrollo tecnológico de la región siendo un proyecto innovador implementado en la única planta de este tipo en la ciudad de Neiva, y además facilita el enriquecimiento académico para sus realizadores, ya que se ven reflejados conocimientos en las diferentes etapas que conllevan a la implementación de este, fomentando mayor experiencia para sus vidas como profesionales.

\section{Metodología}

El diseño del sistema de control computarizado en la planta La Esmeralda permite monitorear y controlar en tiempo real el nivel del tanque de almacenamiento y a partir de este controlar el volumen de agua requerido por el cliente. El diseño se compone de tres etapas, la etapa de recolección de información y selección del medio de comunicación a través de la visita a la planta física, la etapa de instrumentación y control y la etapa de visualización y toma de datos.

\subsection{Recolección de información}

Esta etapa consiste en estudiar y describir la planta La Esmeralda teniendo en cuenta los componentes con los que cuenta para su funcionamiento. La planta La Esmeralda tiene un tanque elevadizo con las siguientes dimensiones, 5.70 metros de largo, por 3.02 metros de ancho y 1.70 metros de alto; pero el mayor nivel de agua está a 1.55 metros de altura ya que a partir de esta altura posee una salida de rebose; y está en la capacidad de almacenar 26.68 metros cúbicos de agua que son alrededor de 7048 galones de agua. La planta La Esmeralda cuenta también con dos pozos cada uno de 7 metros de profundidad, los cuales son los encargados del suministro de agua potable que es bombeada por medio de dos bombas sumergibles de 3 pulgadas de caudal que llenan el tanque elevadizo, además para el proceso de descargue de agua por gravedad cuenta con dos válvulas de salida, cada una de 3 pulgadas de diámetro. Conociendo ya los componentes observados en la visita a la planta física nos encaminamos al estudio posterior de selección del tipo de instrumentos que mejor se ajusten a las características anteriores.

En la figura 1 se observa la planta física La Esmeralda en su estado inicial, es decir sin automatizar, totalmente manual.

El funcionamiento de la planta La Esmeralda antes de su automatización se describe como un sistema mecánico el cual se compone de algunos elementos, dentro de ellos el tanque de almacenamiento descrito a continuación. Es una planta totalmente manual, sin ningún 


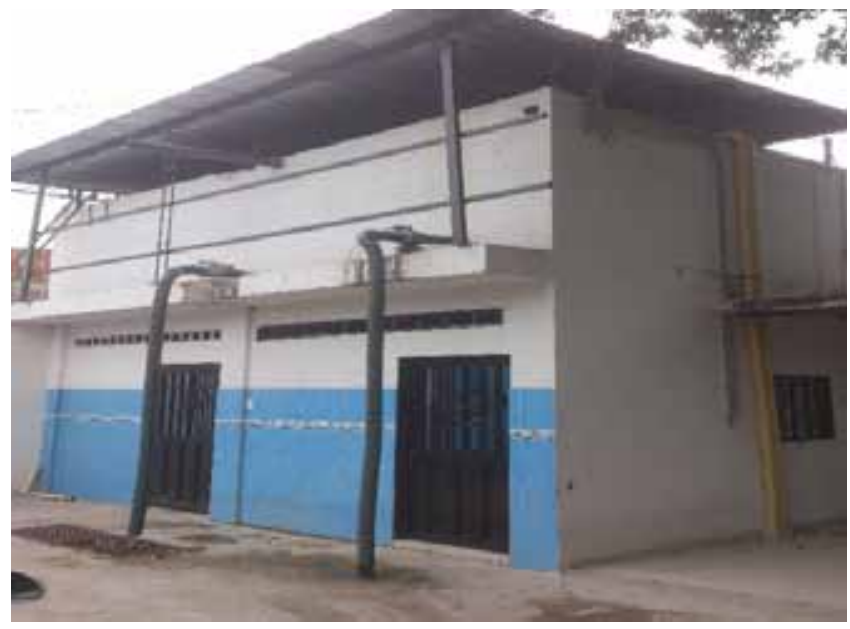

Figura 1. Planta física La Esmeralda.

elemento de medición de nivel, sin ningún tipo de instrumentación electrónica ni sistema de cómputo.

\subsection{Tanque de almacenamiento}

Es una estructura hecha en cemento de forma cubica que es usada para almacenar agua potable a presión ambiente con unas medidas de $1.70 \mathrm{~m}$ de alto, $3.02 \mathrm{~m}$ de ancho y $5.70 \mathrm{~m}$ de largo; es decir que tiene la capacidad de almacenar 7048 galones; consta de dos canales de entrada, dos canales de salida y un canal de rebose. Figura 2.

\subsection{Determinar el sistema de comunicación}

Para el diseño del sistema de comunicación se consideran las características del medio, los diferentes parámetros de operación de las variables a controlar y la relación costo beneficio que hacen que el siste-

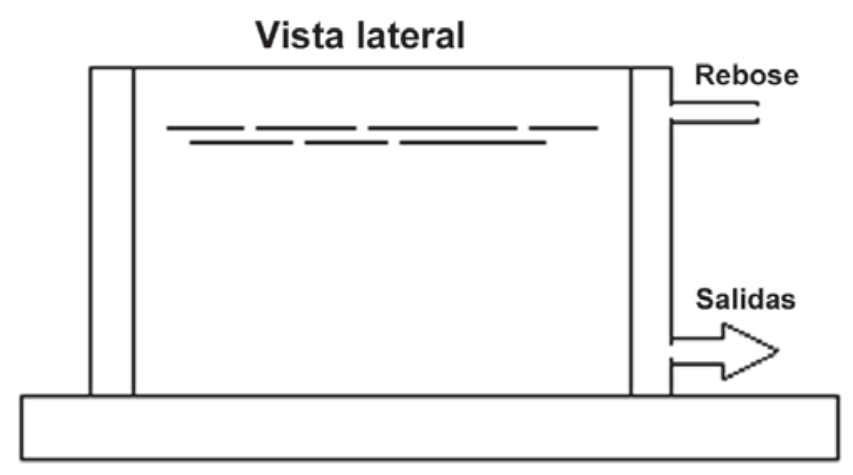

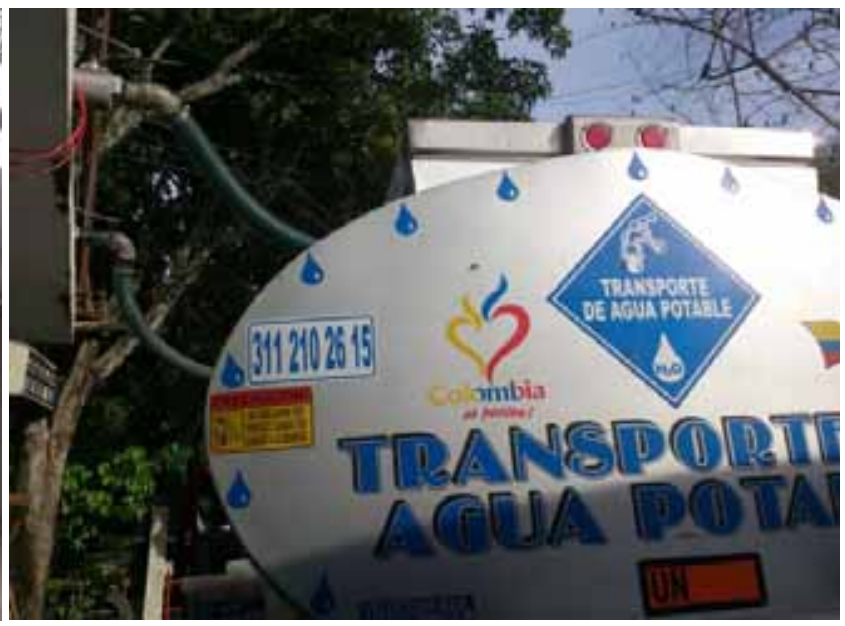

ma seleccionado se ajuste sin ningún problema al desarrollo del proyecto, se emplea un sistema cableado el cual permite lograr el intercambio de señales eléctricas de un punto a otro, ya que el centro de cómputo, el sensor y la tarjeta de control se ubican a una distancia mínima a 5 metros entre ellos, obteniendo así una comunicación sin interferencia y sin pérdida de información.

\subsection{Etapa de Instrumentación y control}

La etapa de instrumentación y control se encarga de la adecuación y adquisición de las variables del proceso, el control de nivel de agua en el tanque elevadizo y la comunicación bidireccional con la etapa de visualización y toma de datos. Lo primero que se busca es obtener la magnitud de la variable del proceso suministrada por el sensor ultrasónico de nivel, posteriormente la adecuación de la señal para obtener niveles

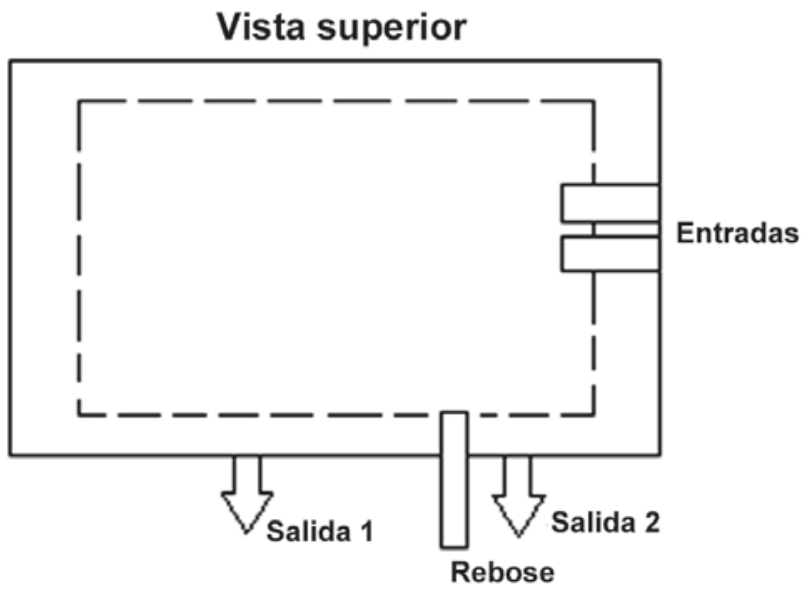

Figura 2. Tanque de almacenamiento. 
de tensión adecuados para su futura adquisición. Luego que los datos son adquiridos se procesan obteniendo el valor real del nivel de agua en el tanque para ser transmitidos al computador para su visualización en la interfaz gráfica de la herramienta LabVIEW. Cabe destacar que el controlador recibe el valor de requerimiento establecido por el cliente desde la interfaz gráfica, realiza el procesamiento de este requerimiento para conocer si el tanque de almacenamiento se encuentra con la disponibilidad de entrega del mismo, para luego tomar la decisión de descargue o por el contrario llenado del tanque para cumplir con el requerimiento y su posterior descargue.

Durante la etapa de instrumentación del proceso se busca seleccionar el sensor que mejor se ajuste a los requerimientos del medio, una vez realizada la selección del sensor que se va a utilizar en este caso el sensor ultrasónico HC-SR04, se procede a realizar el acondicionamiento electrónico necesario para obtener los niveles de tensión adecuados en la entrada del PIC (microcontrolador) logrando con ello una adecuada adquisición de datos para su futuro procesamiento.

El controlador ON/OFF tiene como objetivo controlar dos procesos importantes: primero la apertura y cierre de válvulas de salida o de descargue del tanque elevadizo y segundo el encendido y apagado de las dos bombas sumergibles de tres pulgadas encargadas del suministro de agua potable y llenado del tanque elevadizo.

\subsubsection{Apertura y cierre de válvulas de salida.}

Para la apertura y cierre de las válvulas el controlador recibe el valor del requerimiento de galones de agua solicitado por el cliente enviado desde la interfaz gráfica en labVIEW, compara el valor solicitado con la cantidad de galones de agua disponible en el tanque de almacenamiento elevadizo, posteriormente si el valor solicitado se encuentra por debajo de la existencia de agua en el tanque, automáticamente pone en alto un pin de salida del PIC con el objeto de abrir las válvulas de descargue hasta que la cantidad de galones de agua descargados llegue al valor del requerimiento que el cliente ingresó, para luego cerrar las válvulas y dejarlas en su estado inicial.

\subsubsection{Encendido y apagado de las bombas sumergibles.}

Para el encendido y apagado de las bombas, el controlador ON /OFF funciona a partir del requerimiento solicitado por el cliente, es decir, el cliente solicita un valor determinado de galones de agua para su suministro, este valor viaja desde la interfaz gráfica en labVIEW hasta el controlador, ya en el controlador en la etapa de procesamiento se verifica si el requerimiento del cliente se encuentra o no disponible para su descargue, si el requerimiento no está completo, el controlador no da la orden de apertura de válvulas, de lo contrario las mantiene cerradas y ahora el pin que se pone en alto es el programado para el encendido de las bombas el cual está en alto hasta que el requerimiento esté disponible y listo para ser descargado, en este momento el controlador apaga las bombas e inmediatamente después activa el pin de apertura de válvulas para su descargue.

Luego de culminar el proceso de descargue requerido por el cliente el controlador se dispone a la espera de un nuevo requerimiento para reiniciar el proceso.

\subsection{Etapa de visualización y toma de datos}

La etapa de visualización y toma de datos tiene como objetivos principales el establecimiento de la comunicación bidireccional con la etapa de instrumentación y control, la visualización en tiempo real del estado o nivel de agua almacenado en el tanque elevadizo, permitir la entrada de datos o en nuestro caso del valor del requerimiento en galones de agua por parte del cliente y además se encarga de la toma de datos y registro del usuario que hizo el requerimiento para posteriormente guardar toda esta información en una base de datos que puede ser visualizada y así lograr llevar un registro tanto de la contabilidad como del desempeño y productividad de la planta.

\section{Resultados}

\subsection{Diagrama en bloques del proyecto (Figura 3)}

\subsection{Desarrollo del software requerido para el sistema de control}

Se implementa el software que por medio de la interfaz gráfica LabVIEW de tal forma que incluye el control, la visualización, la adquisición y el almacenamiento de los datos obtenidos de las variables a trabajar, facilitando el manejo y mejorando el medio de operación de la planta, también se desarrolla la debida programación para el PIC18f2550 que conjuntamente con la interfaz gráfica realizan un sistema de control computarizado logrando así la automatización de la planta La Esmeralda. 


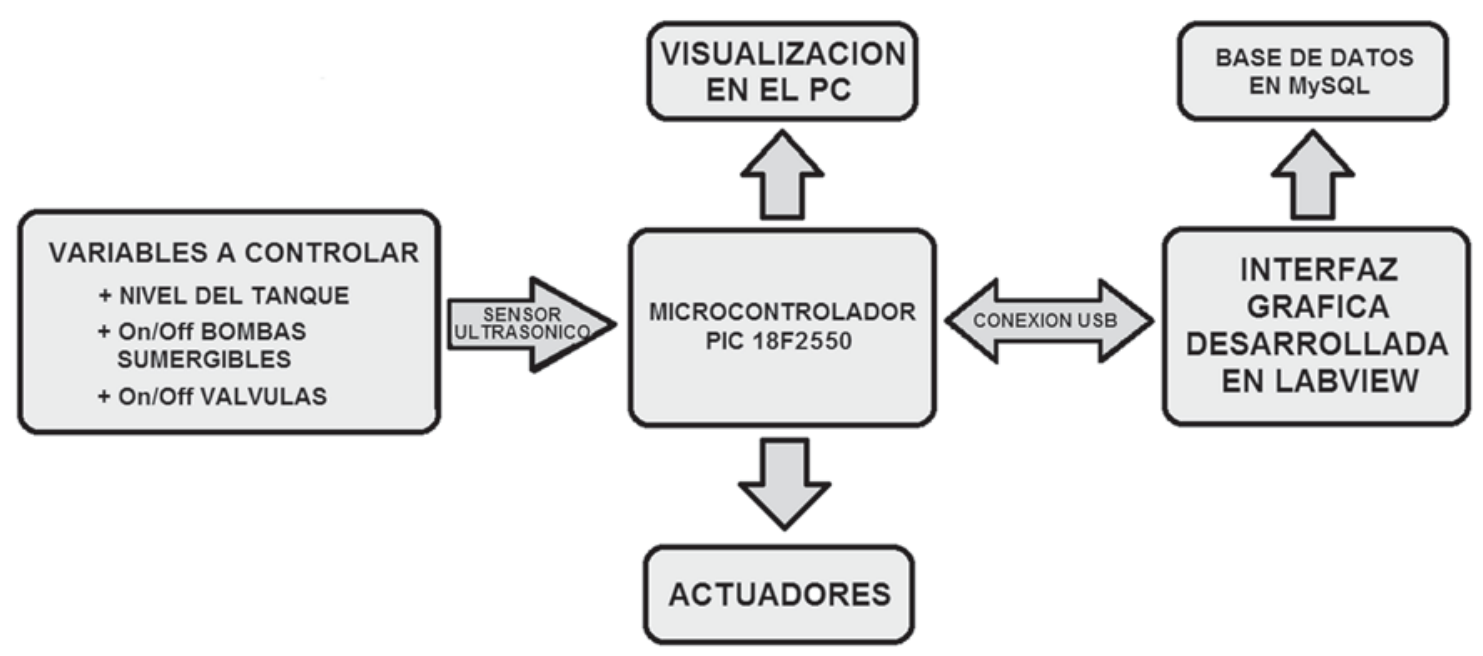

Figura 3. Diagrama de bloques del proyecto

\subsubsection{Interfaz gráfica LabVIEW.}

Para una mejor interpretación acerca del funcionamiento de la interfaz gráfica de control y monitoreo se detallan sus controles y funciones en varios bloques, no sin antes mostrar la interfaz completa, es decir, el Panel de Control mostrado en la Figura 4.

\subsubsection{Bloque de visualización del tanque en tiempo real}

Este bloque se encuentra ubicado en la "Página 1" del panel de control, en él se visualiza el nivel del tanque en tiempo real, posee un indicador numérico en la parte superior que muestra el nivel del tanque en centímetros, además cuenta con tres indicadores luminosos que dejan ver tres estados actuales del nivel del tanque: mínimo, medio y máximo. Ala derecha del panel de control un pequeño bloque cuenta con tres indicadores más, los cuales muestran el volumen de agua almacenado en galones, barriles y metros cúbicos de agua, muy útiles para conocer la cantidad de agua con los que la planta cuenta en cualquier instante de tiempo para su distribución. Finalmente, tres indicadores circulares dan a conocer el estado on/off de las bombas sumergibles y de las electroválvulas del sistema neumático utilizadas para la apertura y cierre de las válvulas (salida del tanque). Figura 5.

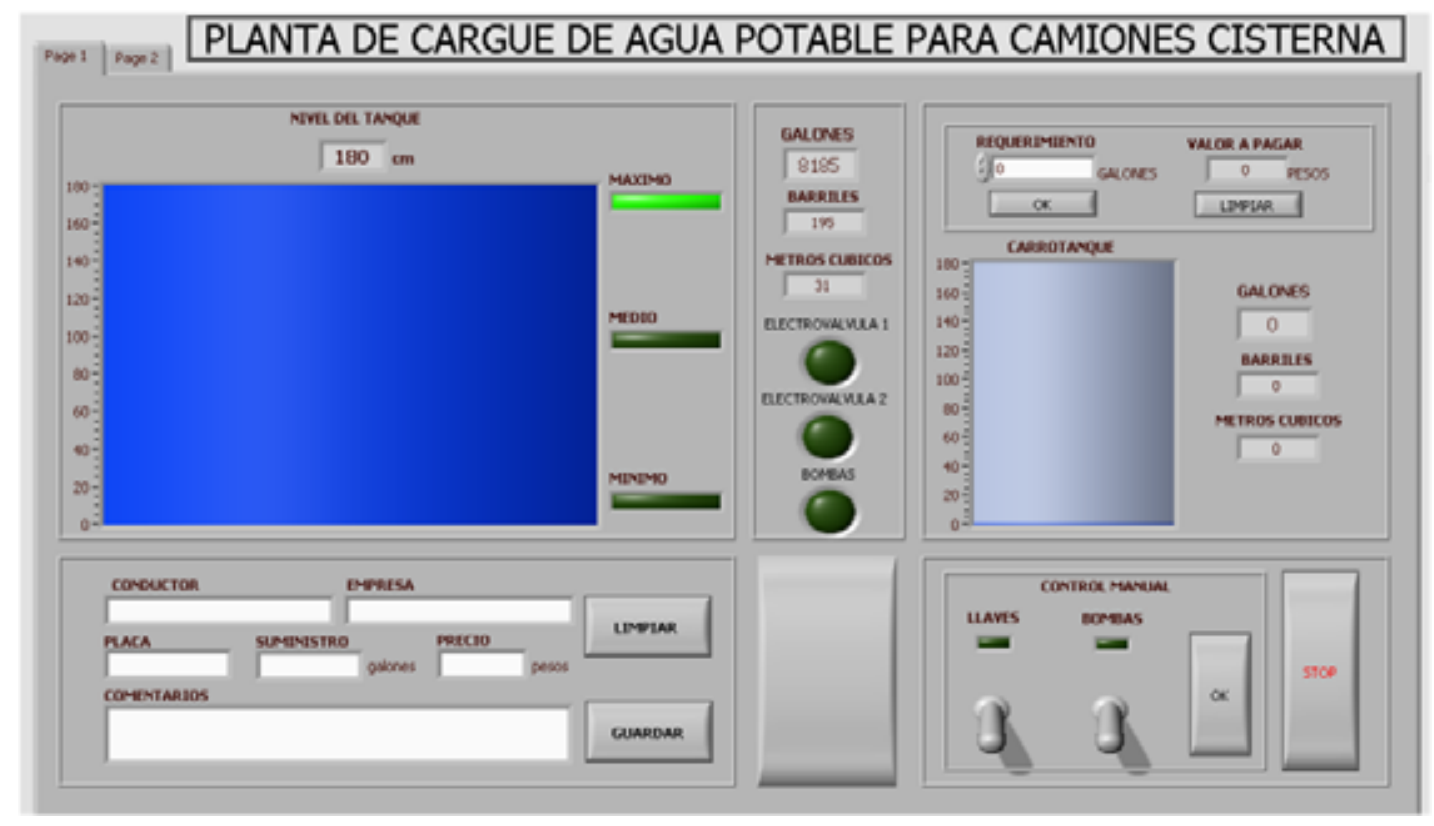

Figura 4. Página 1 del panel de control 


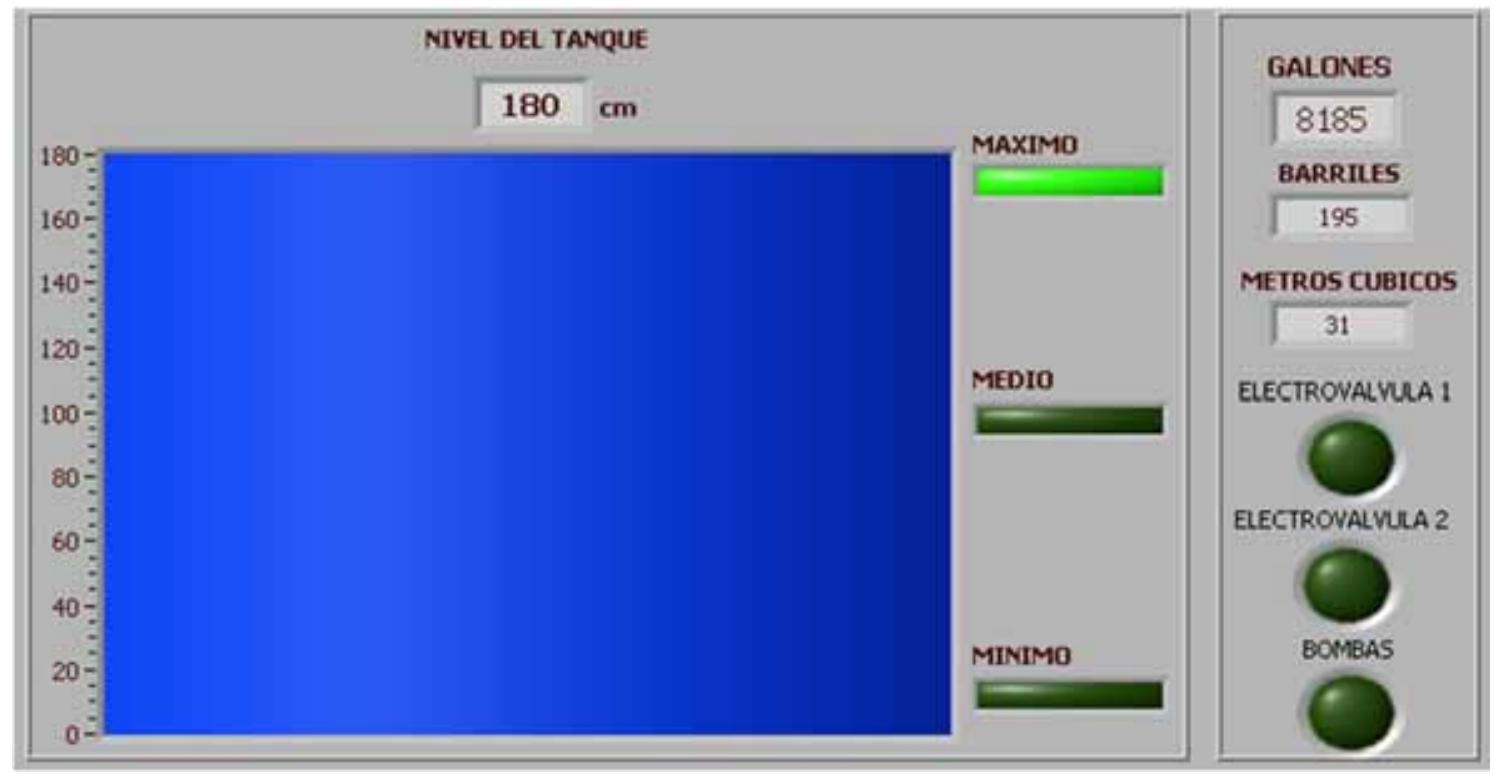

Figura 5. Bloque indicador del nivel del tanque en tiempo real

\subsubsection{Bloque de petición y visualización del requerimiento}

Aquí el operador puede ingresar el valor numérico de galones requeridos por el cliente, una vez ingresado el valor se inicia la descarga del agua requerida dando click en el botón “ok”. Un indicador muestra automáticamente el valor en pesos que el cliente debe cancelar. En la parte inferior un tanque indicador muestra la cantidad de agua que va siendo descargada del tanque principal al camión cisterna que se está cargando y finalmente, para llevar un control visual del agua suministrada tres indicadores numéricos muestran el volumen de agua entregada en galones, barriles y metros cúbicos. Finalizada la entrega al dar clic en el botón limpiar el sistema queda listo para ingresar un nuevo requerimiento. Figura 6.

\subsubsection{Bloque de registro}

Permite el ingreso de la información de los clientes, llenando los campos respectivos tales como: nombre del conductor, nombre de la empresa a la que pertenece, placa del vehículo camión cisterna, valor numérico del suministro cargado por el cliente, valor en pesos cancelados y un campo para agregar algún comentario a tener en cuenta; para guardar los datos digitados anteriormente es necesario dar click en el botón guardar y ya para finalizar el proceso dar clikc en el botón limpiar, encargado de dejar todos los campos en blanco listos para un nuevo cliente. Figura 7.

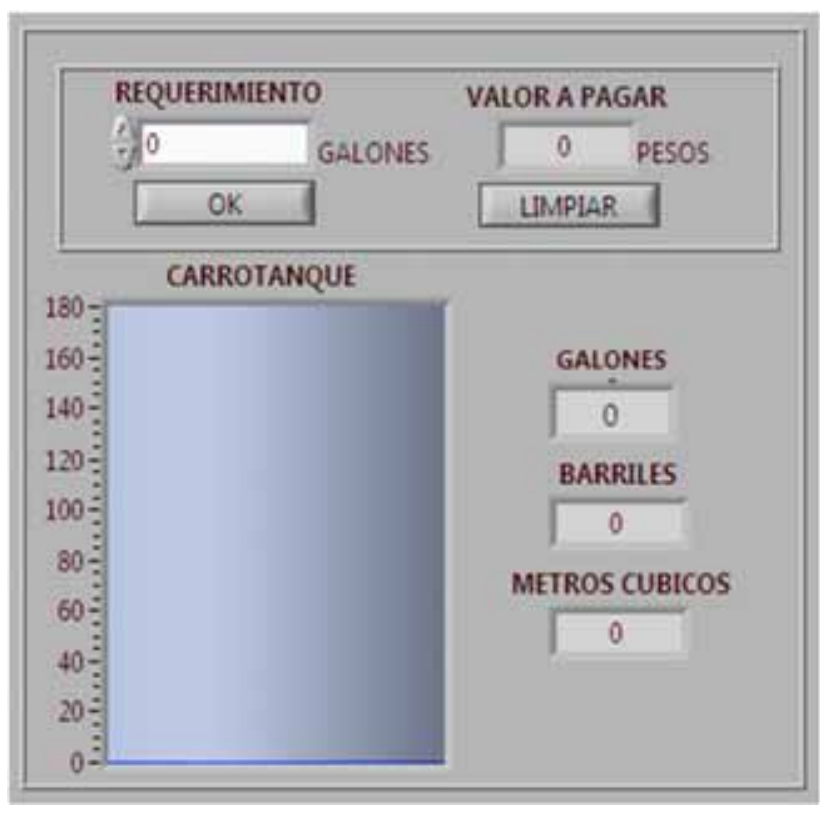

Figura 6. Bloque de petición y visualización del requerimiento

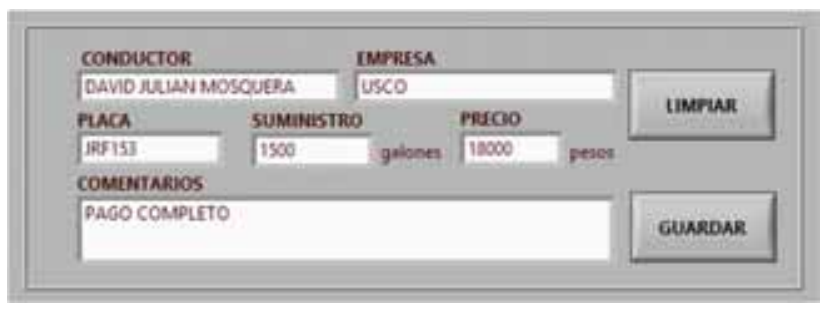

Figura 7. Bloque de registro. 
La visualización de los datos almacenados se puede observar en la "Página 2" del panel de control.

\subsubsection{Bloque de control manual}

Controla la apertura y cierre de las válvulas como el encendido y apagado de las bombas, simplemente cambiando el estado del switch que se seleccione y posteriormente dando clic en el botón ok para iniciar el proceso. También se agrega un botón "stop" que al oprimirlo detiene el proceso de la interfaz. Figura 8.

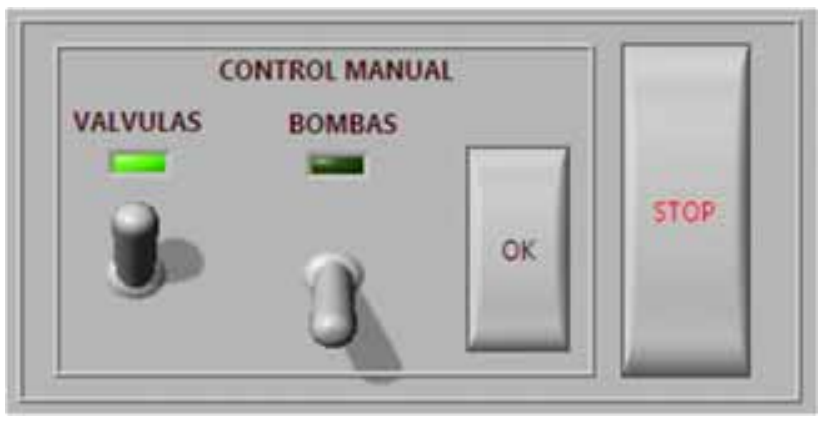

Figura 8. Bloque de control manual.

3.2.1.5 Descripción de la página 2 de la interfaz gráfica diseñada

En esta página se encuentra toda la información consignada en el bloque de registro, la cual permite llevar un control de operación de la planta, donde se observan datos como la fecha/hora, el nombre del conductor del camión cisterna, empresa, placa del vehículo, suministro, precio, y por ultimo algún comentario respecto al servicio prestado. Figura 9.

\subsection{Diseño e implementación del hardware para el sistema de control}

Para el desarrollo de este proyecto se acondiciona el sensor ultrasónico HC-SR04 para la toma en tiempo real del nivel del tanque, para su implementación y ubicación en el tanque se requiere del diseño y desarrollo de una estructura que lo sostiene por encima de la superficie del tanque y lo protege de posibles daños causados por el medio ambiente que lo rodea.

Para el monitoreo del nivel en el tanque se instala el sensor ultrasónico HC-SR04, este sensor trabaja con una alimentación de 5 voltios DC y posee un consumo de corriente menor a $2 \mathrm{~mA}$, está compuesto por un emisor y un receptor. Con el sensor se mide la distancia que hay desde el sensor hasta el nivel de agua en el tanque y lo hace enviando un ultrasonido y midiendo el tiempo en que la señal tarda en regresar, con esta información se calcula la distancia con la ecuación (1). Estos datos son adquiridos por el PIC $18 f 2550$ y enviados a la interfaz gráfica labVIEW para visualizar en tiempo real el nivel del tanque de almacenamiento. Figura 10.

$$
\mathrm{x}=\frac{\mathrm{t} * \mathrm{v}_{\mathrm{s}}}{2} \quad \text { Ecuación (1) }
$$

Donde $x$ representa la distancia, $t$ el tiempo y $v_{s}$ la velocidad del sonido.

La tarjeta diseñada como controlador de la planta cuenta con el PIC 18f 2550 con conexión USB acompañado de la circuitería de amplificación de la señal utilizando opto acopladores 4N25 los cuales funcionan de la mano con transistores 2N3904 y finalmente relés NRP07 que se encargan de alimentar la bobina de las electroválvulas para el funcionamiento del sistema neumático para la apertura y cierre de las válvulas y alimentar las

\begin{tabular}{|c|c|c|c|c|c|c|c|c|}
\hline Dast 1 & Povez 2 & \multicolumn{7}{|c|}{ PLANTA DE CARGUE DE AGUA POTABLE PARA CAMIONES CISTERNA } \\
\hline is & fecherion & conouctor & Bonsa & MACA & Semestio & mon & COMENTAOOSS & 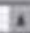 \\
\hline 2 & XV1V/201474337 & meteng & moleper & bould & 2000 & 20000 & swpen efecterey cemsieto & \\
\hline 3 & Q622/2014t23s! & cmar & cons & bowe & 1500 & 6000 & $\operatorname{cose} 20$ & \\
\hline 4 & $2200 / 2013(2005$ & tover & unce & F⿻上丨 & $\$ \infty 0$ & 6000 & $\operatorname{sos}$ & \\
\hline 5 & $1202 / 201521524$ & Musa & Ux<0 & Const2 & 300 & 6000 & DACO COASUTO & \\
\hline 6 & $1202 / 2015) 1931$ & oxus & uxco & $x \times 0.722$ & 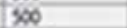 & 6000 & Canctio coesirto & \\
\hline 7 & tearpois jowe & DAVOMOSOUTEA & mavtal & (5)-193 & 200 & 24000 & Daca to00 & \\
\hline 8 & 100200154345 & Aavo & mavtal & 8513 & $\$ \infty 0$ & 6000 & DACO COMEITO & \\
\hline 9 & $1202 / 20155364$ & inen deqe molquers & willocele & sopn-212 & 1500 & 18000 & sase completo & \\
\hline 10 & $2002 / 2015) 224$ & fecer robares & usce & (At5) & 890 & 6000 & $\sec 6$ & \\
\hline 11 & COO1201520335 & IUWOECOMOSCUEA & METLILS & xotis & $1 \times 00$ & 11000 & Carceio & \\
\hline
\end{tabular}

Figura 9. Página 2 de la interfaz gráfica diseñada. 


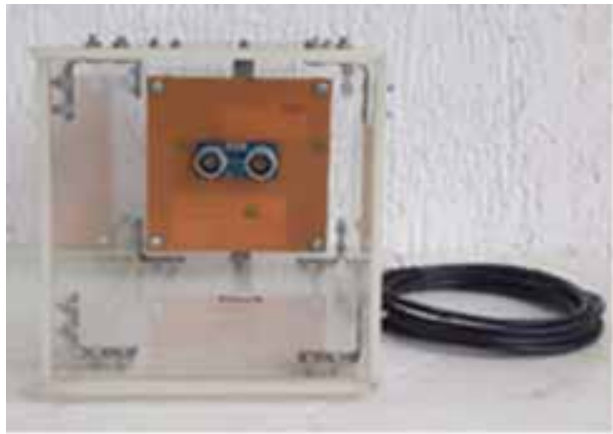

VISTA FRONTAL

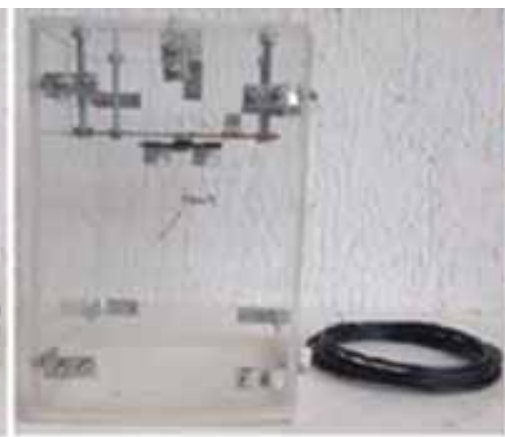

VISTA LATERAL

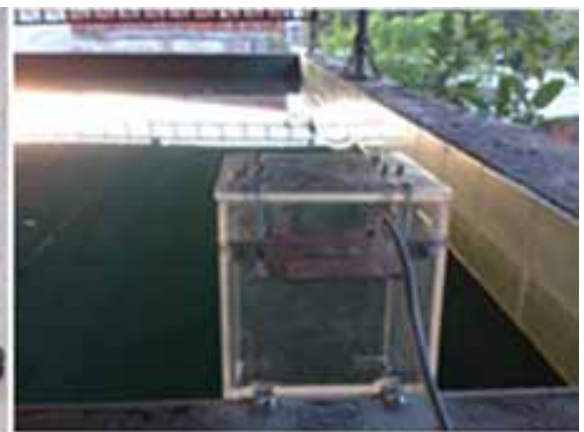

IMPLEMENTACIÓN EN LA PLANTA

Figura 10. Adecuación del sensor ultrasónico HC-SR04.

bobinas de los relés que controlan los contactores de las bombas sumergibles. Esta tarjeta se diseña y se acopla a una caja de acrílico ubicada en el centro de cómputo. Figuras 11 y 12.

La tarjeta de control está compuesta por dos etapas, la etapa de adquisición de datos y procesamiento de información y la etapa de potencia. Para la etapa de adquisición de datos y procesamiento de información el encargado de realizar dichos procedimientos es el PIC $18 f 2550$ y en la etapa de potencia los encargados del encendido y apagado de las bombas, apertura y cierre de las válvulas, son los opto acopladores, transistores, resistencias y relés. Figura 13.

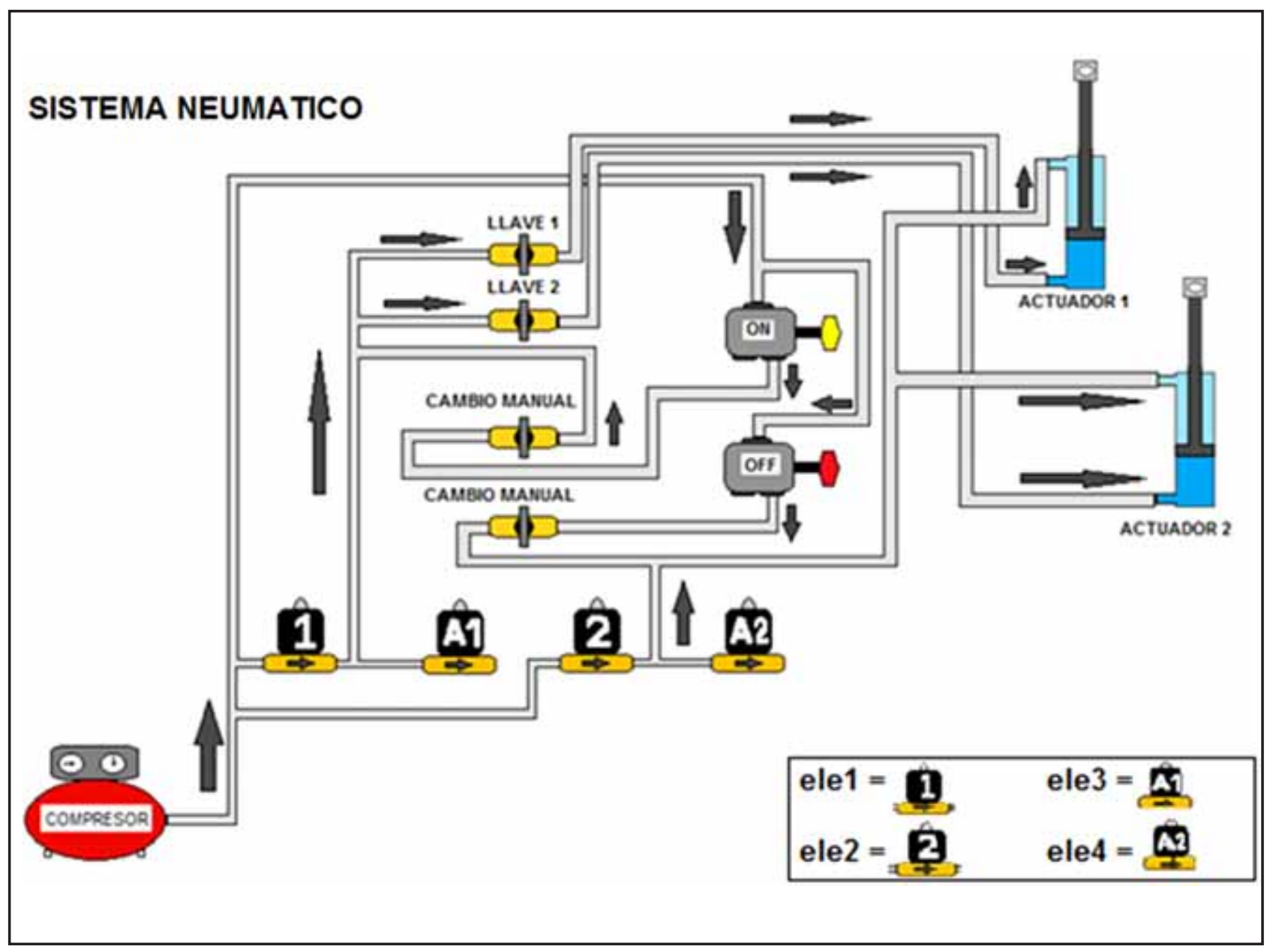

Figura 11. Representación esquemática del sistema neumático. 


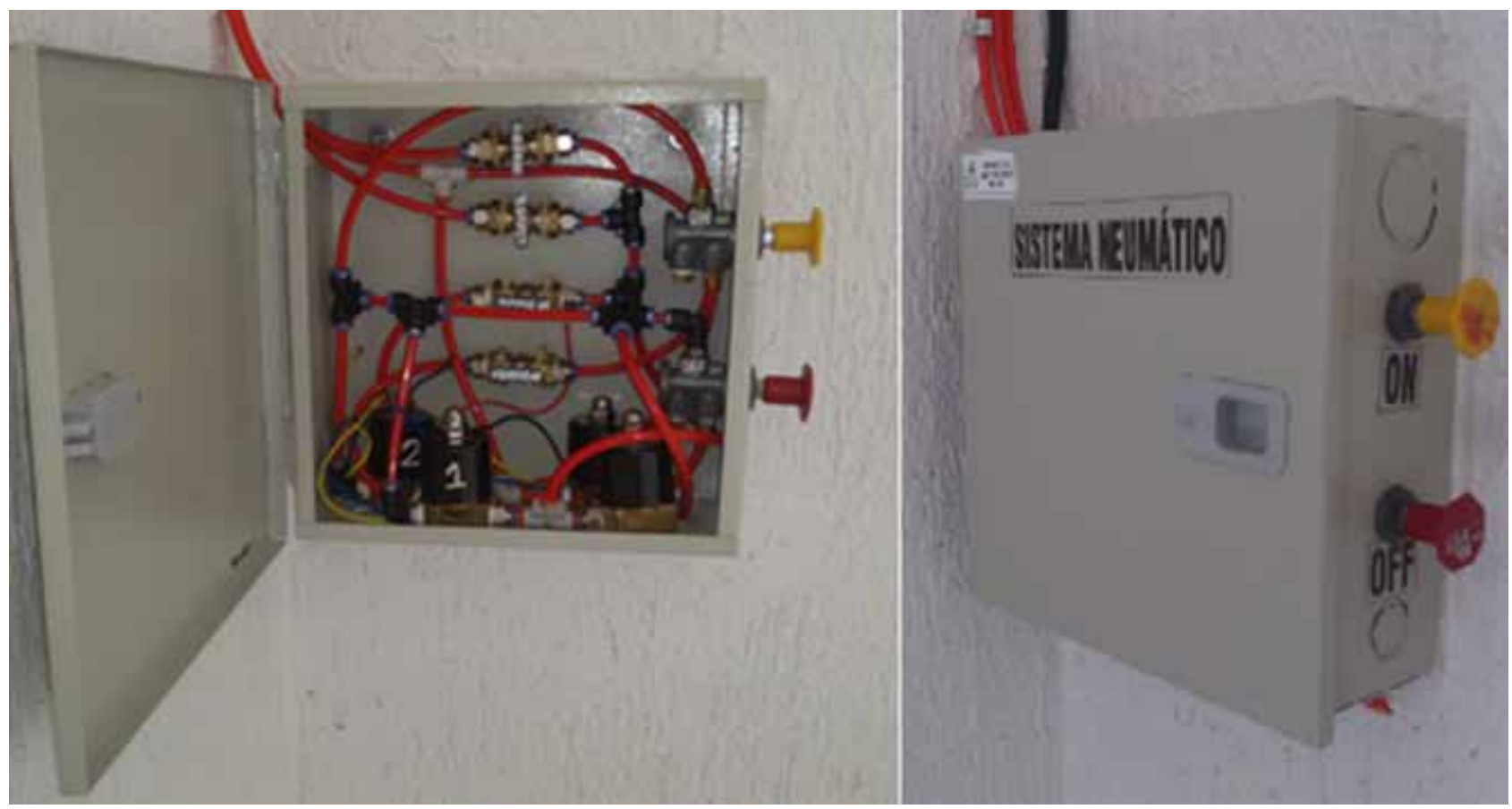

Figura 12. Tablero de control de sistema neumático.
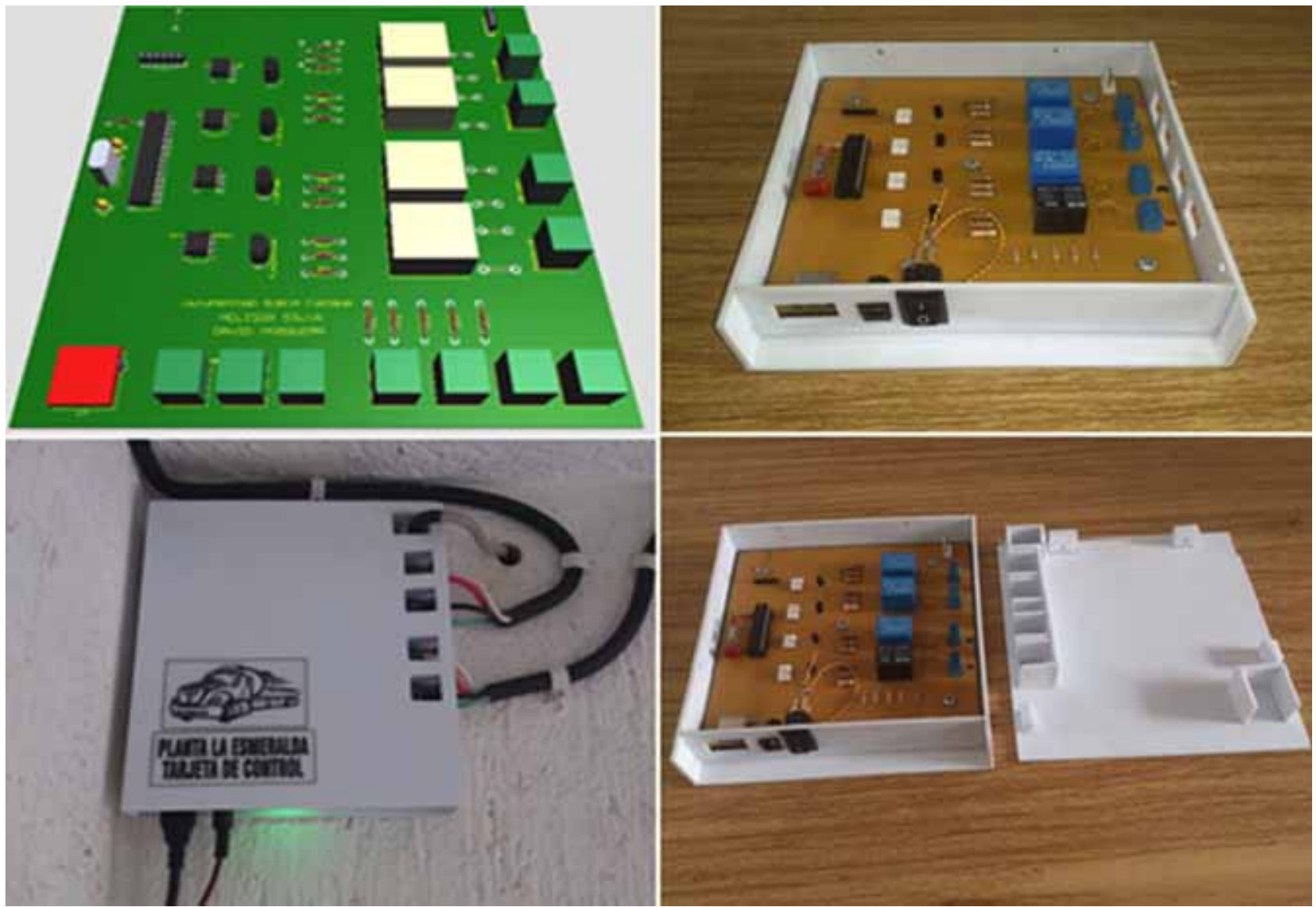

Figura 13. Tarjeta de control. 


\section{Conclusiones}

Se diseñó e implementó un sistema de control computarizado para la planta de cargue de agua potable para camiones cisterna capaz de controlar el nivel del tanque y la cantidad de agua requerida por el cliente. La implementación del proyecto garantiza que el requerimiento solicitado por el cliente sea el deseado evitando perdidas ya sea para el propietario de la planta "La Esmeralda" como para el mismo cliente.

Gracias a las herramientas que nos ofrece LabVIEW logramos desarrollar una interfaz gráfica amigable con el operador capaz de controlar todo el funcionamiento de la planta y además brinda la opción de crear una base de datos con toda la información de los usuarios para llevar a cabo un mejor análisis de la productividad de la planta.

El sistema de control de nivel implementado garantiza que el tanque de la planta La Esmeralda nunca estará vacío ni habrán perdidas de galones de agua por rebose avalando así su correcto funcionamiento.

Para la realización de este proyecto se aplicaron diferentes áreas de la ingeniería electrónica contribuyendo al desarrollo tecnológico de la región, siendo un proyecto innovador para la ciudad de Neiva y el departamento del Huila.

\section{Referencias bibliográficas}

1. Departamento de prensa de Achema, 2009. La automatizacion de procesos, impulsora de multiples sectores industriales. Disponible en http://www. interempresas.net/Quimica/Articulos/29775-Laautomatizacion-de-procesos-impulsora-de-multiplessectores-industriales.html. Consultado el 11 de agosto de 2014.

2. Camilla, L., 2006. Efectos de la Automatizacion. Consultado el 9 de septiembre de 2014. Disponible en http://www.emb.cl/electroindustria/articulo.mvc? xid $=515 \&$ edi $=9$.

3. Quiminet, 2012. Empresas de automatización. Consultado el 10 de agosto de 2014. Disponible en http: //www.quiminet.com/empresas/empresas-deautomatizacion-2736251.htm.
4. Revista Española de Electrónica, 2014. Tome el control de sus datos con LabVIEW 2014. Consultado el 6 de agosto de 2014. Disponible en http://www. redeweb.com/articulos/articulo.php?inicio=0\&pag $=$ 1\&id=1251.

5. Domínguez, J., 2007. Información en tiempo real del proceso productivo. Técnica Industrial 258.

6. Atlantic International University, 2008. Mantenimiento Industiral. Consultado el 6 de septiembre de 2014. Disponible en https://cursos.aiu.edu/Mantenimiento\%20 Industrial/PDF/Tema\%201.pdf.

7. Kuo, B., 1996. Sistemas de Control Automático. 7 ed. Prentice Hall. 897 páginas.

8. Microchip Technology Inc., 2006. pic18f2550 Data Sheet. Disponible en http:/ww1.microchip.com/ downloads/en/devicedoc/39632c.pdf. Consultado el 16 de agosto de 2014.

9. Ogata, K., 1998. Ingeniería de Control Moderna. 3 ed. Prentice Hall. 1015 páginas.

10. Ospina López, V.H., 2003. Diseño del centro de control para aguas de Manizales. Colombia. (Tesis postgrado). Facultad de ingeniería y arquitectura. Especialista en automatización industrial. Universidad Nacional de Colombia sede Manizales. 75 páginas.

11. Peñaloza Calderón, J.A.; Mora Gómez, S., 2008. Automatización e instrumentación de una planta embotelladora a escala. Colombia. (Tesis pregrado). Ingeniería electrónica. Universidad Pontificia Bolivariana. 131 páginas.

12.Pino Villarroel, J.A., 2009. Propuesta de automatización y control para la planta de inyección de agua salada de la estación de descarga Bared. Barcelona. (Tesis pregrado). Ingeniería de sistemas. Universidad de Oriente. 122 páginas.

13. Roncancio, H. A.; Cifuentes, H., Tutorial de Lab VIEW. Universidad Distrital Francisco José de Caldas. Laboratorio de Electrónica. 100 páginas.

14.Ultra Plas S.A. de C.V. 2012. Propiedades del acrílico. Obtenido de acrilico y policarbonato.com: Disponible en http://www.acrilico-y-policarbonato.com/ acrilico-propiedades.html. Consultado el 12 de septiembre de 2014. 